\title{
Osteogenic commitment of Wharton's jelly mesenchymal stromal cells: mechanisms and implications for bioprocess development and clinical application
}

Raquel Cabrera-Pérez ${ }^{1,2^{*}}$, Marta Monguió-Tortajada ${ }^{3}$, Ana Gámez-Valero ${ }^{3}$, Raquel Rojas-Márquez ${ }^{1,4}$, Francesc Enric Borràs ${ }^{3,5}$, Santiago Roura ${ }^{6}$ and Joaquim Vives ${ }^{1,2,7^{*}}$ (D)

\begin{abstract}
Background: Orthopaedic diseases are one of the major targets for regenerative medicine. In this context, Wharton's jelly (WJ) is an alternative source to bone marrow (BM) for allogeneic transplantation since its isolation does not require an invasive procedure for cell collection and does not raise major ethical concerns. However, the osteogenic capacity of human WJ-derived multipotent mesenchymal stromal cells (MSC) remains unclear.

Methods: Here, we compared the baseline osteogenic potential of MSC from WJ and BM cell sources by cytological staining, quantitative real-time PCR and proteomic analysis, and assessed chemical and biological strategies for priming undifferentiated WJ-MSC. Concretely, different inhibitors/activators of the TGF $31-B M P 2$ signalling pathway as well as the secretome of differentiating BM-MSC were tested.

Results: Cytochemical staining as well as gene expression and proteomic analysis revealed that osteogenic commitment was poor in WJ-MSC. However, stimulation of the BMP2 pathway with BMP2 plus tanshinone IIA and the addition of extracellular vesicles or protein-enriched preparations from differentiating BM-MSC enhanced WJ-MSC osteogenesis. Furthermore, greater outcome was obtained with the use of conditioned media from differentiating BM-MSC.
\end{abstract}

Conclusions: Altogether, our results point to the use of master banks of WJ-MSC as a valuable alternative to BM-MSC for orthopaedic conditions.

Keywords: Mesenchymal stromal cells, Bone marrow, Wharton's jelly, Osteogenic differentiation, Bone regeneration

\section{Background}

The development of new treatments for bone-related diseases resulting from trauma or pathophysiological age-, sex- or infection-associated bone resorption has become a priority in the field of regenerative medicine [1-4]. In this context, autologous cell-based therapy has been presented as a promising approach to promote bone regeneration in both pre-clinical and clinical settings [5-8]. However, their clinical translation needs the delivery of safe and efficacious products, which can be largely hampered by age and comorbidities of the cell donor [9-13]. In contrast, allogeneic

\footnotetext{
*Correspondence: rcabrera@bst.cat; jvives@bst.cat

'Cell Therapy Service, Blood and Tissue Bank (BST), Barcelona, Catalonia, Spain

Full list of author information is available at the end of the article
}

off-the-shelf cell products derived from healthy and immune-compatible donors are very attractive since they are immediately available and provide a high number of cells $[14,15]$.

Multipotent mesenchymal stromal cells (MSC) constitute a heterogeneous population of non-haematopoietic multipotent cells which can be isolated from a variety of human body sources [3, 16, 17]. In particular, MSC have a fibroblast-like appearance, plastic adherence, the ability to differentiate into tissues of mesodermal lineages (adipocytes, chondrocytes and osteocytes) and a specific cell surface expression pattern, according to the minimal criteria established by the International Society for Cell and Gene Therapy (ISCT) [18]. Of note, a couple of MSCbased products have already received marketing approval

(C) The Author(s). 2019 Open Access This article is distributed under the terms of the Creative Commons Attribution 4.0 International License (http://creativecommons.org/licenses/by/4.0/), which permits unrestricted use, distribution, and 
[19], whereas most developments are still in clinical evaluation, including MSC-based tissue engineering products (EudraCT Nos. 2010-024041-78, 2010-023998$18,2010-023999-12$ and 2013-005025-23) under development in our laboratory [20].

Bone marrow (BM) has become the most used source of MSC in the orthopaedic field because of its intrinsic osteogenic differentiation potential, but alternative sources are garnering attraction. These include Wharton's jelly (WJ), which is the connective tissue surrounding the human umbilical cord and is advantageous for cell collection since its isolation is not painful, does not require invasive procedures and does not raise major ethical concerns [21]. Accordingly, we reported the feasibility of expanding clinical-grade WJMSC from samples typically discarded from public cord blood banking programmes [22]. Moreover, in contrast to adult BM-MSC, WJ-MSC are expected to be more primitive, proliferative and immunosuppressive cells, particularly for the lack of HLA-DR antigens [23-26]. Nevertheless, the osteogenic capacity of WJ-MSC remains under scrutiny.

Osteogenic differentiation of mesenchymal precursors and bone regeneration are extremely complex processes regulated by the interaction of different signalling pathways including TGF $\beta / B M P$, MAPK, Wnt, Hedgehog, Notch and AKT/mTOR [27]. Among them, the TGF $/$ BMP pathway plays the major role in the regulation of osteoblast lineagespecific differentiation, bone induction, maintenance and repair and constitutes a promising target for the treatment of bone diseases [28-30]. Up to date, about 60 TGF $\beta$ family proteins have been identified so far, being TGF $\beta 1$ and BMP2 ligands the most widely investigated due to their positive role in bone formation in vivo [31, 32]. However, while BMP proteins have been demonstrated to induce the expression of MSC differentiation factors (such as DLX5 and $R U N X 2)[33,34]$, several in vitro studies have described a negative impact of TGF $\beta 1$ on the terminal differentiation of osteoblast precursors [34-36].

Despite several reports investigating the osteogenic capabilities of BM- and WJ-MSC have been published to date, it is still difficult to integrate existing data due to heterogeneity in MSC isolation and culture procedures. In the present study, we aimed to provide homogeneous and comparative data regarding the ability of BM- and WJ-MSC to differentiate towards the osteogenic lineage by means of cytological staining and molecular and proteomic analysis. Moreover, we evaluated a variety of strategies based on the modulation of the TGF $\beta /$ BMP pathway and the use of the BM-MSC secretome to enhance osteogenesis in WJ-MSC and emulate BM-MSC osteogenic commitment.

\section{Methods}

\section{Cell culture}

BM-MSC $(n=3)$ and WJ-MSC $(n=3)$ (passage 3-5) were isolated according to 'Good Manufacturing Practice for Advanced Therapy Medicinal Products' (GMP for ATMPs, European Commission Guidelines of 2017.11.22) and further expanded in Dulbecco's modified Eagle's medium (DMEM) (31885-023; Gibco) containing $2 \mathrm{mM}$ glutamine and supplemented with 10\% human serum B (hSerB)-'expansion medium' [37, 38]. All cell cultures were maintained at $37^{\circ} \mathrm{C}$ and $5 \% \mathrm{CO}_{2}$ in humidified incubators, and media were changed every 3-4 days. Cell number and viability were determined by the haemocytometerbased trypan blue dye exclusion assay.

\section{Phenotype assessment}

Immunophenotypic characterisation of MSC was performed using the following antibodies: mouse anti-human CD45-fluorescein isothiocyanate (CD45-FITC) (Clone HI30; 555482; BD Pharmingen), anti-human CD105phycoerythrin (CD105-PE) (Clone 43A4E1; 130-117-696; Miltenyi Biotec), anti-human HLA-DR-FITC (Clone L243; 347363; BD Biosciences), anti-human CD90-PE (Clone F15-42-1-5; IM1840U; Beckman Coulter), anti-human CD31-FITC (Clone WM59; 555445; BD Pharmingen) and anti-human CD73-PE (Clone AD2; 550257; BD Pharmingen). Cells were stained for $15 \mathrm{~min}$ at room temperature (RT), washed and re-suspended in phosphate-buffered saline (PBS) (14190-094; Gibco) as described elsewhere [38]. Acquisition and data analysis were performed using a FACSCalibur cytometer and the CellQuest Pro software (Becton Dickinson), respectively.

\section{Osteogenic differentiation assays}

BM-MSC and WJ-MSC (passage 3-5) were seeded until $70-80 \%$ confluence $\left(10^{4}\right.$ and $2 \times 10^{4}$ cells $/ \mathrm{cm}^{2}$, respectively). 'Differentiation media' composed of the StemPro osteogenesis differentiation kit (A1007201; Gibco) supplemented with 100 units $/ \mathrm{mL}$ of penicillin and $100 \mu \mathrm{g} /$ $\mathrm{mL}$ streptomycin (Penicillin-Streptomycin; P4458; Sigma-Aldrich) was used for the osteogenic induction in vitro. Alkaline phosphatase (ALP) (B5655; SigmaAldrich) and alizarin red (AR) (2003999; Merck Millipore) staining were finally carried out to assess cell differentiation.

\section{Gene expression assays}

Total RNA was purified from cell cultures using the RNeasy Plus Mini Kit (74134; Qiagen) according to the manufacturer's instructions, quantified using NanoDrop Lite (Thermo Scientific), and electrophoresed in $1 \%$ agarose gels to confirm integrity and purity. cDNA synthesis was then performed by reverse-transcription PCR (RT-PCR) using the High-Capacity cDNA Reverse Transcription Kit (4368814; Thermo Fisher Scientific) according to the manufacturer's instructions. cDNA was finally amplified by quantitative real-time PCR (qRTPCR) using the TaqMan gene expression assays listed in 
Table 1. In all cases, target gene expression was referred to $G A P D H$ expression by using the $2^{-\Delta \mathrm{Ct}}$ method.

\section{Inhibition/activation of TGF $\beta / B M P 2$ signalling pathways}

WJ-MSC (passage 4) were seeded at a cell density of $2 \times$ $10^{4}$ cells $/ \mathrm{cm}^{2}$. Inhibition of the TGF $\beta$ signalling pathway was carried out by addition of galunisertib (LY2157299) (sc-391123; Santa Cruz Biotechnology) to the osteogenic differentiation media at a final concentration of $10 \mu \mathrm{M}$. For BMP2 pathway stimulation, human recombinant BMP2 (SRP6155; Sigma-Aldrich) and/or tanshinone IIA (sc-200932; Santa Cruz Biotechnology) were added to a final concentration of $100 \mathrm{ng} / \mathrm{mL}$ and $5 \mu \mathrm{M}$, respectively. All compounds were added immediately after every media change after 1 week of in vitro osteogenic induction with differentiation media.

\section{Extracellular vesicle isolation and characterisation}

To avoid sample contamination with exogenous extracellular vesicles (EVs), cells were cultured in EV-depleted media. For EV depletion, $2 \times$ differentiation media was ultra-centrifuged at $100,000 \times g$ for $\geq 16 \mathrm{~h}$ and diluted with StemPro Basal Media (A10069-01; Gibco) to 1× working concentration.

Supernatants derived from undifferentiated BM-MSC and WJ-MSC (passage 3-5) or from BM-MSC under osteogenic differentiation (passage 3-5) were collected at weeks $0,1,2$ and 3 , and were sequentially centrifuged at $400 \times g$ for $5 \mathrm{~min}$ and at $2000 \times g$ for $10 \mathrm{~min}$ to exclude cells and cell debris. Conditioned medium (CM) was then concentrated by $100-\mathrm{kDa}$ ultrafiltration using Amicon Ultra (UFC910024; Millipore) at $2000 \times g$ for 35 min, obtaining typically $250 \mu \mathrm{L}$ of concentrated CM (CCM). EVs were isolated by size exclusion chromatography (SEC) as previously reported [39]. Protein elution was checked by reading absorbance at $280 \mathrm{~nm}$ using NanoDrop (Thermo Scientific).

The presence of EVs in the SEC fractions was determined according to the presence of tetraspanins by bead-based flow cytometry [39]. Briefly, EVs were coupled to $4-\mu \mathrm{m}$

Table 1 TaqMan gene expression assay ID

\begin{tabular}{ll}
\hline Gene & Assay ID \\
\hline MSX2 & Hs00741177_m1 \\
DLX5 & Hs01573641_mH \\
RUNX2 & Hs01047973_m1 \\
SP7 & Hs05049492_s1 \\
ALPL & Hs01029144_m1 \\
TFGB1 & Hs00998133_m1 \\
BGLAP & Hs01587814_g1 \\
COLIA2 & Hs01028970_m1 \\
GAPDH & Hs02786624_g1 \\
\hline
\end{tabular}

aldehyde/sulphate-latex microspheres (A37304; Invitrogen) for $15 \mathrm{~min}$ at RT and blocked in BCB buffer (PBS supplemented with $0.1 \%$ BSA (A4503) and 0.01\% $\mathrm{NaN}_{3}$ (S8032); Sigma-Aldrich) on overnight rotation. EV-coated beads were spun down at $2000 \times g$ for 10 min, washed with $B C B$ buffer and re-suspended in PBS. EV-coated beads were labelled with the primary antibodies anti-CD9 (Clone VJ1/ 20) and anti-CD63 (Clone TEA3/18) (kindly provided by M. Yáñez-Mó (CBM-SO, IIS-IP, UAM, Madrid, Spain) and F. Sánchez-Madrid (Hospital Universitario de la Princesa, IIS-IP, UAM, CNIC, Madrid, Spain)) or the IgG isotype control (a637355; Abcam) and secondary antibody FITCconjugated Goat $\mathrm{F}\left(\mathrm{ab}^{\prime}\right) 2$ Anti-Mouse IgG (1032-02; Bionova). EV-coupled beads were washed after each step with BCB buffer and centrifuged at $2000 \times g$ for $10 \mathrm{~min}$. Data was acquired in a FACSLyric flow cytometer (BD) and analysed by FlowJo v.X software (Tree Star).

SEC-EV-containing fractions were examined for EV size and morphology by cryo-electron microscopy (cryo-EM). Vitrified specimens were prepared by placing $3 \mu \mathrm{L}$ of a sam-

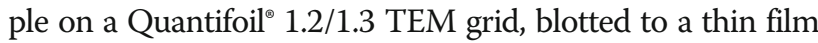
and plunged into liquid ethane- $2_{(1)}$ in the Leica EM CPC cryoworkstation (Leica). The grids were transferred to a 626 Gatan cryoholder and maintained at $-179^{\circ} \mathrm{C}$. Samples were analysed with a Jeol JEM-2011 transmission electron microscope (Jeol) operating at an accelerating voltage of $200 \mathrm{kV}$. Images were recorded on a Gatan UltraScan 2000 cooled charge-coupled device (CCD) camera with the DigitalMicrograph software package (Gatan).

\section{Proteomic analysis}

The protein content of EV-enriched fractions was analysed by liquid chromatography followed by mass spectrometry (LC-MS/MS) on Orbitrap XL (Thermo Fisher) for three independent undifferentiated cultures for each MSC type. Data was searched against the Swiss-Prot human database (downloaded in August 2016), using the search algorithm Mascot v2.5.1. Only peptides showing a false discovery rate (FDR) lower than 5\% were retained. Proteins identified with at least two unique peptides and found in all three samples were considered for further analysis.

The obtained proteomic profile for our samples was compared with previous studies compiled in EV-specific databases EVpedia [40], ExoCarta [41] and Vesiclepedia [42].

\section{Data analysis}

Statistical analysis was performed with the GraphPad Prism 6 software (GraphPad Software, Inc.). Descriptive data were expressed as mean \pm standard deviation (SD). Multiple $t$ tests were used for investigating differences between BM- and WJ-MSC at different time points along the osteogenic differentiation. Statistical significance was set at $" p<0.05$ and ${ }^{* *} p<0.01$. 


\section{Results}

WJ-MSC exhibit delayed osteogenic induction compared with BM-MSC

BM-MSC and WJ-MSC were highly positive for CD73, CD90 and CD105 and negative for CD31, CD45 and HLA-DR expression according to the ISCT criteria (Additional file 1).

The osteogenic differentiation of BM-MSC and WJMSC cultures were assessed by alkaline phosphatase (ALP) and alizarin red (AR) staining. All BM-MSC and WJ-MSC cell lines showed osteogenic potential in vitro after osteogenic induction with specific differentiation media. However, a delay in osteogenesis was observed in WJ-MSC compared to BM-MSC. In particular, as shown in Fig. 1a, most of the cells in BM-MSC cultures displayed a marked baseline activity of the osteogenic marker ALP. In contrast, in WJ-MSC cultures, only few cells exhibited ALP activity even at week 5. Regarding the results obtained for AR staining, calcium depositions were clearly visible in BM-MSC cultures at week 3 . However, 5 weeks were required in order to obtain similar results in WJ-MSC (Fig. 1b).

\section{BM-MSC show increased expression of osteogenic markers compared to WJ-MSC}

We further analysed the delayed osteogenesis in WJ-MSC cultures. For that purpose, we comparatively assessed the time-course expression of key osteogenic genes implicated in MSC differentiation. Interestingly, we found some significant differences along both BM- and WJ-MSC osteogenic differentiation. Regarding osteogenic transcription factors (Fig. 2a), BM-MSC showed a progressive increase in DLX5, RUNX2 and SP7 expression. In contrast, in WJMSC, DLX5 expression was gradually decreased and SP7 levels exhibited an increment from week 2 to 5 . Additional differences were observed in MSX2. Remarkably, a steady increase of $M S X 2$ expression was observed up to week 5 in WJ-MSC, whereas no changes in MSX2 expression were detected in BM-MSC.

Moreover, the expression patterns of main early and late osteogenic markers were comparatively assessed (Fig. 2b). In this sense, the early marker $A L P L$ reached its maximum expression level during the first week in BM-MSC. However, in WJ-MSC, ALPL expression was reduced at this time compared to week 0 and started to

\section{A}
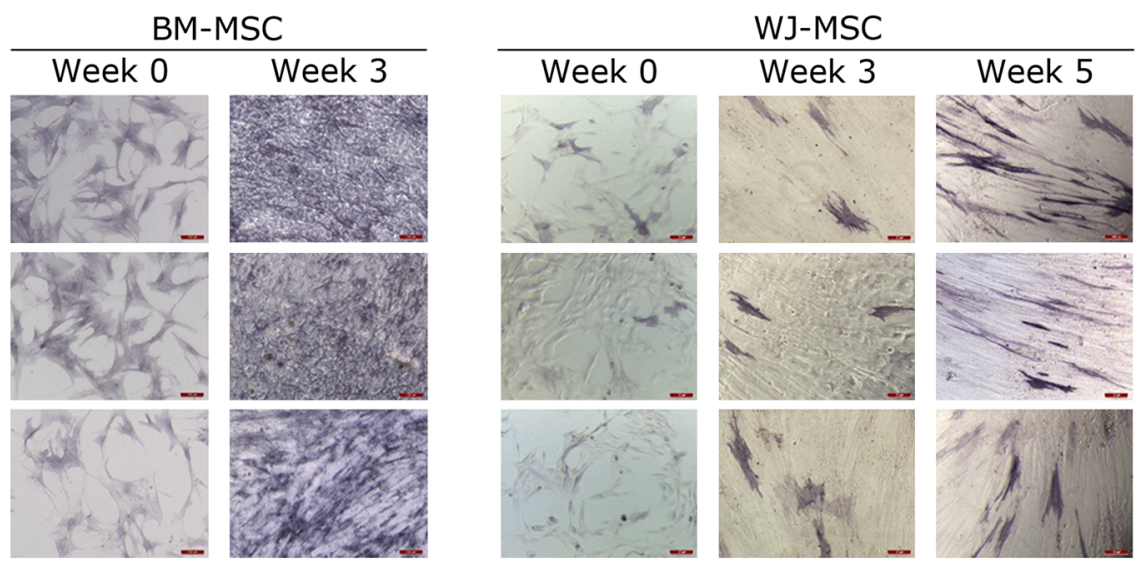

B

BM-MSC
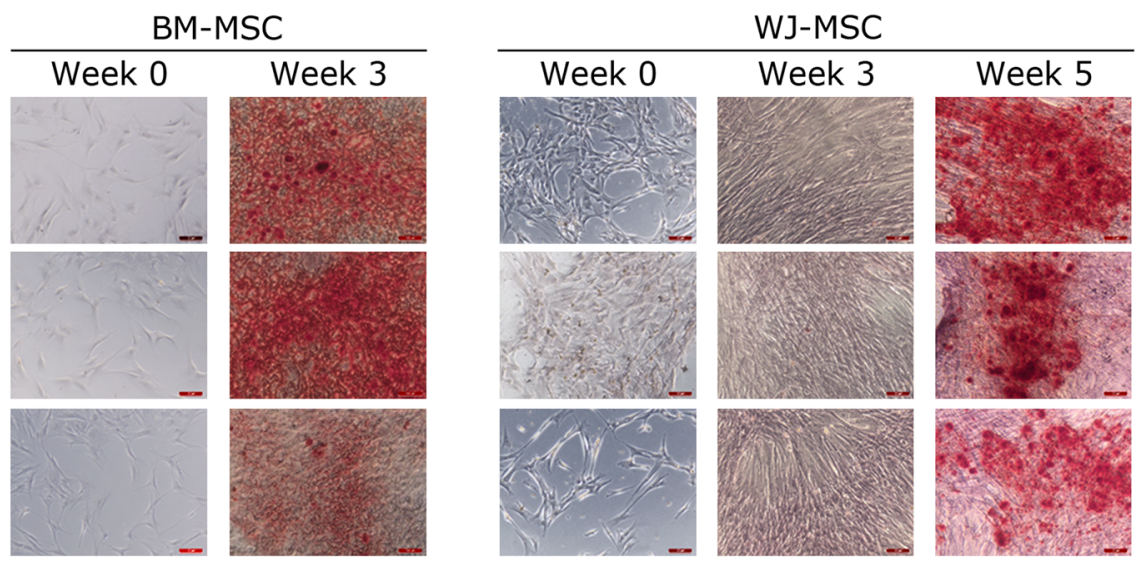

Fig. 1 Differences in timing for osteogenic differentiation in BM-MSC and WJ-MSC cultures. Alkaline phosphatase (a) and alizarin red (b) staining at indicated times. $N=3$ for both MSC types. Scale bars: $100 \mu \mathrm{m}$ 


\section{A}

MSX2

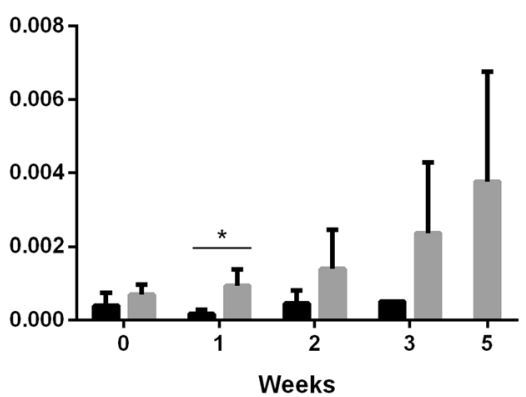

RUNX2

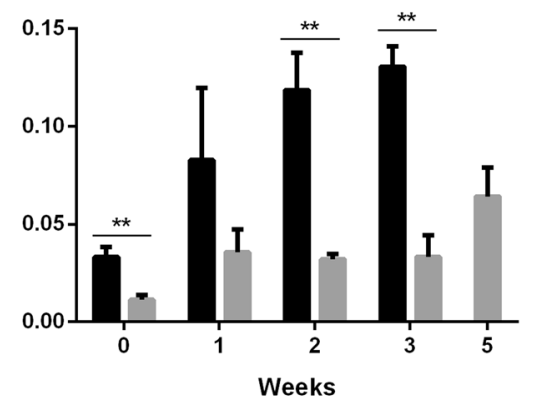

B

ALPL

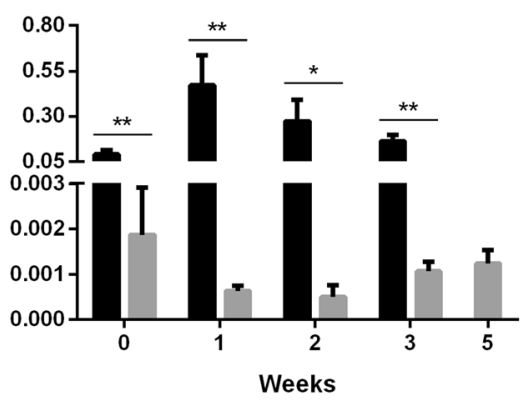

BGLAP

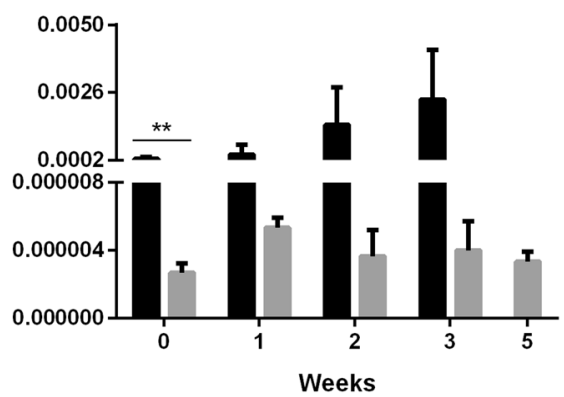

BM-MSC

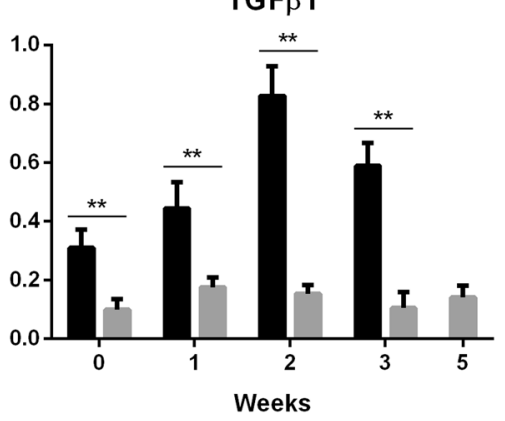

COL1A2

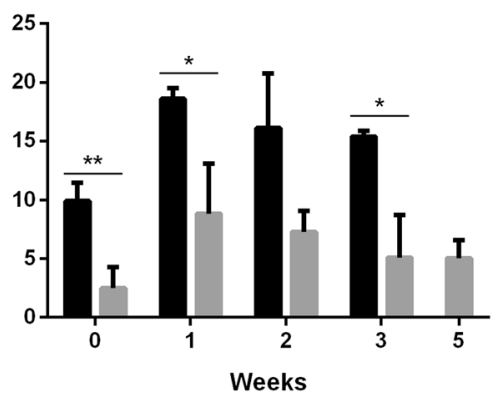

WJ-MSC

Fig. 2 Gene expression profiles of the main markers involved in osteogenic differentiation. Expression levels of osteogenic transcription factors (a) and early/late osteogenic markers (b). Bars represent mean \pm SD. ${ }^{*} p<0.05$ and ${ }^{* *} p<0.01$ (multiple $t$ tests). $N=3$ for each MSC type 
increase again from the third week. The expression patterns of late osteogenic markers TGF $\beta 1$ and BGLAP were also different. In BM-MSC, TGF $\beta 1$ achieved the highest expression level at week 2 and BGLAP expression increased progressively. On the contrary, no changes were observed for these genes in WJ-MSC. In regard to COL1A2, similar expression patterns were obtained for both cell types, although expression in BM-MSC was twofold higher than that in WJ-MSC. Remarkably, the expression of $R U N X 2$, DLX5, ALPL, TGFB1, BGLAP and COL1A2 was promoted in BM-MSC even when they were in an undifferentiated stage. Taken together, these findings indicate a higher osteogenic differentiation commitment in BM-MSC.

\section{Promotion of BMP2 signalling primes osteogenic differentiation of WJ-MSC}

Subsequently, the role of TGF $\beta 1$ and BMP2 signalling pathways in the promotion of osteogenic differentiation of WJ-MSC was investigated. To prevent TGF $\beta$ activation, differentiation media was supplemented from week 1 to 3 with galunisertib. On the other hand, in order to stimulate osteogenic differentiation through the BMP2 signalling pathway, human recombinant BMP2 and/or the BMP activator tanshinone IIA were also added to the differentiation media (Fig. 3a).
As shown in Fig. 3b, the inhibition of TGF $\beta 1$ signalling had no effect on WJ-MSC osteogenesis. Similarly, negative results in AR staining experiments were seen when BMP2 and tanshinone IIA were present alone or in combination with galunisertib. In contrast, when BMP2 and tanshinone IIA were added together, calcium depositions were clearly visible in WJ-MSC cultures after 3-week induction, emulating the behaviour of BM-MSC under standard osteogenic inducing conditions. This suggests the promotion of WJ-MSC osteogenic differentiation following stimulation of BMP2 signalling.

\section{BM-MSC-conditioned media strongly enhances WJ-MSC osteogenic differentiation}

The results obtained after the characterisation of the gene expression profiles of some of the main osteogenic markers explained, in part, the superior osteogenic potential presented by BM-MSC. However, with the aim of deepening in the elucidation of the increased osteogenic capacity shown by BM-MSC, the proteomic content associated with the isolated extracellular vesicles (EVs) from the supernatant of undifferentiated BM-MSC and WJ-MSC cultures was analysed. Proteomic analysis by LC-MS/MS allowed the identification of several EV markers, such as annexin A2, A5 and A6, glyceraldehyde-3-phosphate dehydrogenase and CD5L. As depicted in Fig. 4, 99 proteins were

A

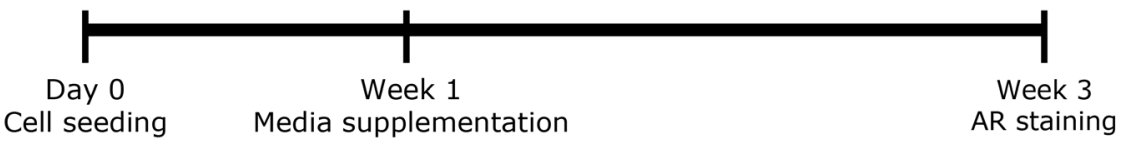

B

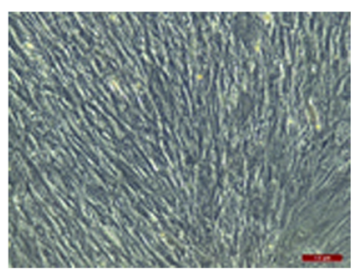

Galunisertib $10 \mu \mathrm{M}$

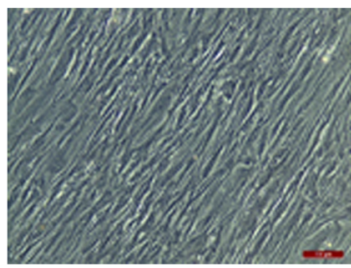

Galunisertib $10 \mu \mathrm{M}$

BMP2 $100 \mathrm{ng} / \mathrm{ml}$

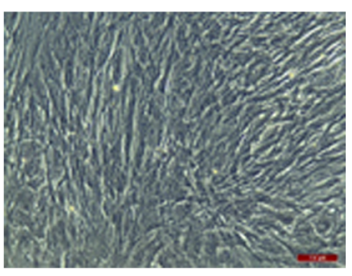

BMP2 $100 \mathrm{ng} / \mathrm{ml}$

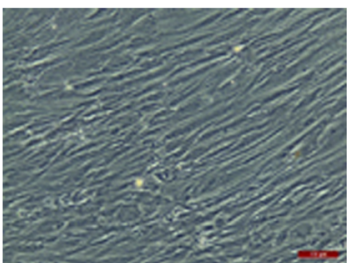

Galunisertib $10 \mu \mathrm{M}$ Tanshinone IIA $5 \mu \mathrm{M}$

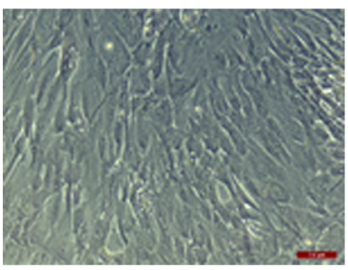

Tanshinone IIA $5 \mu \mathrm{M}$

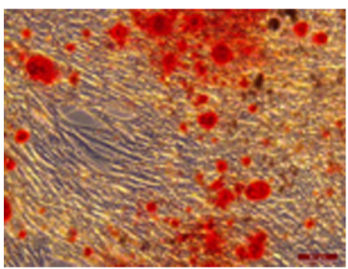

BMP2 $100 \mathrm{ng} / \mathrm{ml}$ Tanshinone IIA $5 \mu \mathrm{M}$

Fig. 3 Modulation of the TGF $\beta / B M P$ signalling pathway to stimulate WJ-MSC osteogenic differentiation. a Scheme of the experimental design. From day 0 to week 1, cells were cultured in osteogenic differentiation media, which was supplemented from week 1 to 3 with galunisertib, BMP2 and/or tanshinone IIA. b Representative AR staining results obtained in passage 4 WJ-MSC after 2 weeks of culture in osteogenic media supplemented with galunisertib $10 \mu \mathrm{M}, \mathrm{BMP} 2100 \mathrm{ng} / \mathrm{mL}$ and tanshinone lla $5 \mu \mathrm{M}$ in different combinations. Scale bars: $100 \mu \mathrm{m}$ 


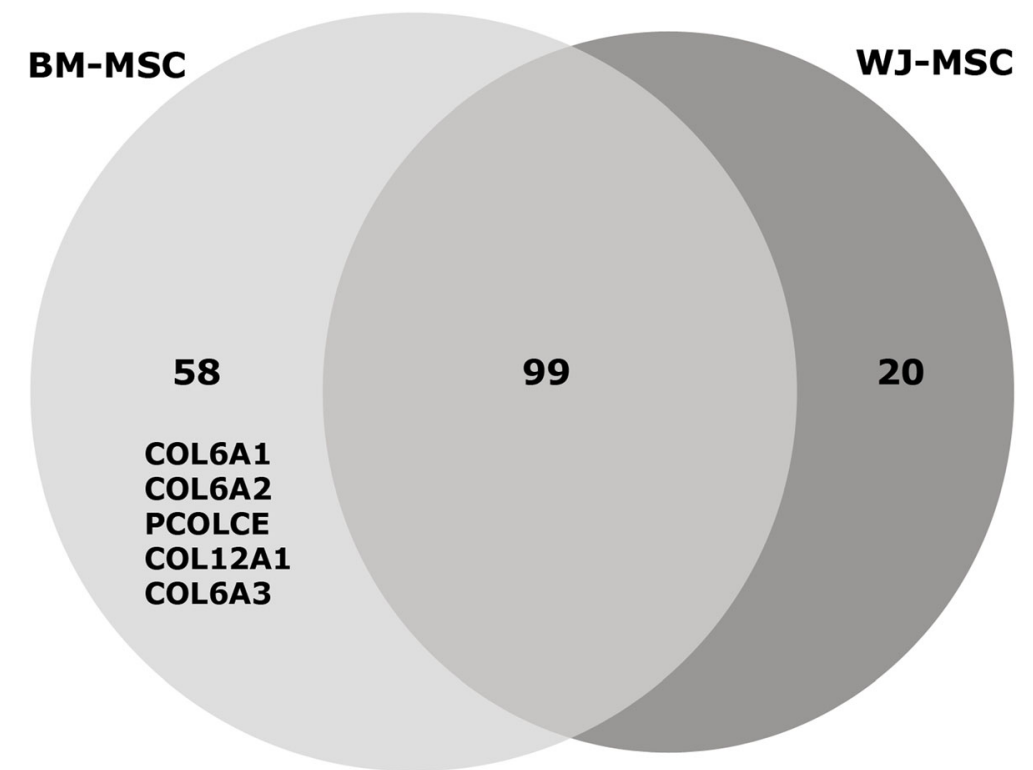

Fig. 4 Osteogenic markers found in undifferentiated BM-MSC- and WJ-MSC-secreted EVs. Number of proteins found in extracellular vesicles (EVs) isolated by size exclusion chromatography ( $n=3$ different cell lines for BM-MSC and WJ-MSC). The osteogenic markers differentially overrepresented in BM-MSC EV samples are indicated. Results were obtained by LC-MS/MS, two peptides restricted, FDR $<5 \%$

found in common between both types of cells. However, five osteogenic markers (namely COL6A1, COL6A2, PCO LCE, COL12A1 and COL6A3) were differentially overrepresented in BM-derived EVs compared to WJ-MSC EVs. This finding could contribute to the explanation of the higher osteogenic commitment observed in BM-MSC and suggested the possibility of using the BM-MSC secretome to prime osteogenesis in WJ-MSC.

To test the feasibility of this approach, conditioned media (CM) from BM-MSC cultures were obtained weekly up to week 3 of the osteogenic differentiation and processed by size exclusion chromatography to separate and purify fractions enriched in EVs or soluble proteins (Additional file 2). Once isolated, the EV or protein fractions were added to WJ-MSC differentiating cultures.

Monitoring of the osteogenic differentiation was performed by AR staining. Negative results were obtained in all cases after 2 weeks of media supplementation with either EVs or protein fractions (data not shown). However, after 3 weeks, calcium depositions were observed in all the WJ-MSC cultures supplemented with BMsecreted EVs and protein fractions purified from BMMSC differentiating cultures at weeks 1, 2 and 3, while the secretome from undifferentiated BM-MSC (week 0) did not (Fig. 5a). Although both EVs and protein fractions positively stimulate WJ-MSC osteogenesis, the effect produced by the soluble protein fraction resulted in a greater outcome than that produced by EVs collected at the same differentiation stage. Based on this observation, the full CM collected from BM-MSC cultures after 1 week of in vitro osteogenic induction was also tested
(Fig. 5b). As shown in Fig. 5b, the addition of CM from differentiating BM-MSC to WJ-MSC cultures resulted in higher osteogenesis than the addition of purified EVs or protein fractions separately. Furthermore, the fact that calcium depositions were clearly identified from the very first week indicated a powerful synergistic positive effect between EV and proteins secreted by BM-MSC in the progression along the osteogenic linage differentiation.

\section{Discussion}

The use of allogeneic MSC is promising for the treatment of bone-related conditions. In this context, some studies have suggested that master cell banks of WJ-MSC may offer advantages over the use of BM-MSC [20, 22]. However, to guarantee the success of WJ-MSC in situations where bone repair or bone regeneration is required, it is essential to demonstrate that osteogenic commitment is equally driven in both BM- and WJ-MSC.

In the present study, we have extensively characterised the osteogenic differentiation process of BM- and WJ-MSC in order to provide homogeneous data because, despite there are many reports in the field describing osteogenic properties of both cell lines, data sets are often incomplete and non-comparable due to heterogeneity in isolation and culture procedures.

In concordance with previous observations reported by other authors in the field [43, 44], our findings evidenced that WJ-MSC are less prone to differentiate into bone cells than BM-MSC. We thus aimed to comparatively analyse the molecular machinery associated with osteogenic differentiation in BM- and WJ-MSC since we 


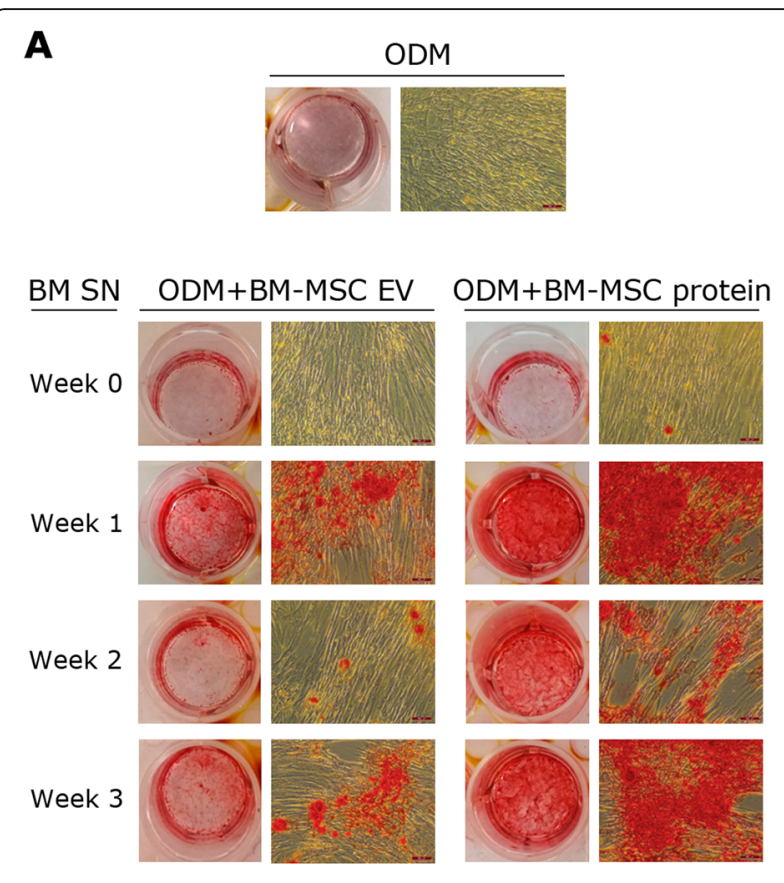

B

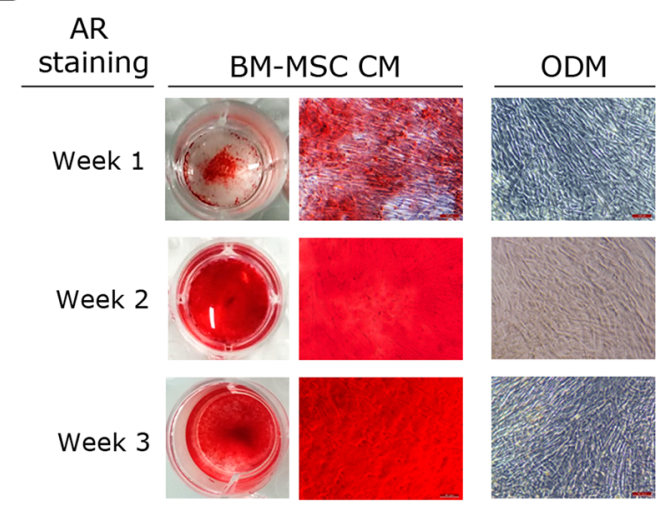

Fig. 5 Effect of BM-MSC-derived products on osteogenic commitment of WJ-MSC. a Representative images of AR staining in passage 4 WJ-MSC after 3 weeks of culture with ODM alone (top) or supplemented with either EV or protein fractions collected from undifferentiated BM-MSC (week 0) or BM-MSC cultures at week 1, 2 or 3 of osteogenic differentiation (bottom). b Representative images of AR staining in passage 4 WJ-MSC after 1, 2 or 3 weeks of culture with CM obtained from differentiating BM-MSC cultures at week 1. CM conditioned media, EV extracellular vesicles, ODM osteogenic differentiation media, SN supernatant. In all cases, scale bars: $100 \mu \mathrm{m}$

found a marked delay in the osteogenic progression of WJ-MSC compared to BM-MSC. Results obtained by qRT-PCR confirmed that BM-MSC exhibit an osteogenic gene profile more similar to that of osteoblast and evidenced that MSX2, a TGF $\beta 1$-induced factor reported to promote cell proliferation and supress osteogenic differentiation by inhibiting DLX5-driven effects [36, 45-48], was overexpressed in WJ-MSC. This suggests that the balance between $M S X 2$ and $D L X 5$ expression could be critical in MSC, resulting in either a proliferating or differentiating outcome.

Trying to revert the scenario found in WJ-MSC, we then attempted to modulate TGF $\beta$ - and BMP-mediated signalling pathways by adding specific activators or inhibitors in order to repress MSX2 and/or to stimulate DLX5 expression. To this end, we used different combinations of chemical factors including (i) galunisertib, a receptor antagonist that specifically targets and binds TGF $\beta$ RI [49]; (ii) BMP2, which has been reported to induce osteoblast differentiation by promoting DLX5 expression [33, 50] and (iii) tanshinone IIA, a phytochemical compound reported to enhance BMP signalling stimulation [51]. Neither the use of each molecule separately nor the combination of a repressing and activating agent resulted in an osteogenic output in WJ-MSC. However, agreeing with the results published by Heo and collaborators in umbilical cord blood MSC [52], the addition of tanshinone IIA to BMP2induced cultures significantly increased calcium depositions in WJ-MSC after 3 weeks, thus matching WJ-MSC osteogenic behaviour to that of their BM-MSC counterparts.

Although systemic infusion of MSC has been shown to increase bone growth and repair in clinical trials [53-55], administered cells engraft poorly. In this line, previous in vivo studies carried out by our group in which ovine $e G F P+$ BM-MSC were infused in an ovine model of osteonecrosis of the femoral head demonstrated the presence of non-stained eGFP osteocytes in newly formed bone matrix, suggesting that contribution of MSC lies also in paracrine signalling that activate and recruit host osteoblasts to the bone repair areas [56]. Increasing evidence have shown that nanosized, membrane-encapsulated EVs are one of the most active MSCs' secreted factors [25]. Indeed, EVs can serve as powerful tools for cell-free therapy due to precise multifunctional molecular cargoes [57, 58]. However, significant differences have been described in the content of EV purified from MSC cultures of different origins. In terms of miRNA profiles, BM-MSC-derived EVs have been described to present a miRNA cargo that is tightly related to MSC differentiation [59]. Furthermore, different profiles of miRNAs have also been reported depending on the differentiation stage of the secretory cell [60]. Here, we studied the differences in the protein content of EVs obtained from undifferentiated BM- and WJ-MSC. As a result, COL6A1, COL6A2, PCOLCE, COL12A1 and COL6A3 osteogenic markers were differentially overrepresented in BM-derived EVs compared to WJ-MSC EVs. Interestingly, COL6 and COL12 interactions have been reported to control and promote bone formation in early phases due to their role in the establishment of matrix bridges between adjacent cells when pre-osteoblasts establish cell-cell communication [61]. These observations prompted us to evaluate the effect of BM-MSC-derived EVs in the osteogenic differentiation of WJ-MSC. Additionally, the impact of the 
soluble protein fractions (eluted in the latter size exclusion chromatography (SEC) fractions) purified from BM-CM was also determined.

Both EV-rich and soluble protein fractions from differentiating BM-MSC cultures promote bone differentiation in WJ-MSC. On the contrary, the secretome of undifferentiated BM-MSC does not have an osteogenic effect. This fact matches the differences reported in the miRNA content of MSC-EVs depending on differentiation stages and explains, in part, the little differences found in the protein content of undifferentiated BMMSC and WJ-MSC EVs. Remarkably, the use of unprocessed CM obtained from differentiating BM-MSC has a greater impact on osteogenic induction and produces not only an exacerbation on the mineralisation of the culture but also a shortening in the differentiation time. This suggests that EV- and protein-rich fractions from differentiating BM-MSC act by different and synergistic pathways, thus pointing out that BM-MSC and WJ-MSC therapeutic efficacy could be equivalent when administered within the bone microenvironment, where BMMSC are present, and mitigating the need to overstimulate WJ-MSC osteogenesis ex vivo. This is particularly relevant due to the implications of WJ-MSC osteogenic priming in manufacturing development and clinical applications which include (i) prolonged cell cultures for ex vivo stimulation; (ii) difficulties of cell trypsinisation once the differentiation process is started; (iii) higher costs associated with the increment in time for cell culture and the use of additional GMP grade products; and (iv) possible clinical complications surrounding the use of priming compounds. Furthermore, the effect associated with the use of $\mathrm{CM}$ could be enhanced in vivo due to the immune response produced under pathophysiological conditions, which positively contributes to bone regeneration.

\section{Conclusions}

Despite their multipotentiality, the intrinsic molecular signature of WJ-MSC described here highly counteracts their osteogenic differentiation and thus their future application in cell-based therapies against orthopaedic conditions. However, our findings demonstrate that secreted factors in the CM from differentiating BM-MSC cultures greatly enhance WJ-MSC osteogenesis and suggest that intra-bony environment could be enough to guarantee WJ-MSC-promoted bone regeneration. This fact avoids the need either to overstimulate WJ-MSC osteogenesis ex vivo or to use genetically modified WJ-MSC. Therefore, although further research is required in order to evaluate the therapeutic benefit of WJ-MSC in the context of orthopaedic diseases, the use of GMP-grade master cell banks of WJ-MSC may be a valuable alternative to those of BM-MSC.

\section{Supplementary information}

Supplementary information accompanies this paper at https://doi.org/10. 1186/s13287-019-1450-3.

Additional file 1 Immunophenotypic characterization of BM-MSC and WJMSC. Boxes represent median and 5-95 percentiles. $N=3$ for each cell type.

Additional file 2. EV and protein fractions isolation and characterization. (A) Scheme of the methodological procedure followed for EV and protein isolation from BM-MSC and WJ-MSC conditioned media. CCM, concentrated conditioned media; CM, conditioned media; EV, extracellular vesicles; SEC, size exclusion chromatography; SN, cell culture supernatant. (B) Example of representative elution profile obtained for CD9 and CD63 EV markers quantification by bead-based flow cytometry (left axis) and for protein elution monitoring by absorption at $280 \mathrm{~nm}$ (right axis) in the different SEC fractions. MFI, mean fluorescence intensity. (C) Cryo-EM images confirming EVs presence in pooled EV fractions. Scale bar: $200 \mathrm{~nm}$.

\section{Abbreviations}

ALP: Alkaline phosphatase; AR: Alizarin red; BM: Bone marrow; CM: Conditioned media; EV: Extracellular vesicle; FDR: False discovery rate; GMP: Good manufacturing practice; ISCT: International Society for Cell and Gene Therapy; MSC: Mesenchymal stromal cell; ODM: Osteogenic differentiation media; qRT-PCR: Quantitative real-time PCR; RT: Room temperature; SEC: Size exclusion chromatography; WJ: Wharton's jelly

\section{Acknowledgements}

The authors would like to acknowledge Clémentine Mirabel and Blanca Reyes for the technical support and advice, and Dr. Kristel van Landuyt for critically reviewing the manuscript and providing helpful comments.

\section{Authors' contributions}

RC-P, FEB, SR and JV conceived the study. RC-P, MM-T, AG-V and RR-M performed the experiments and analysed the data. RC-P wrote the manuscript. All authors revised the manuscript. All authors approved the final version of the manuscript.

\section{Funding}

Work in our laboratory is supported by the Spanish Cell Therapy Network (TerCel, expedient No. RD16/0011/0028 and RD16/00111/0006), Ministerio de Ciencia Innovación y Universidades de España (Instituto de Salud Carlos III, expedient No. Pl19/01788), Fundació la Marató de TV3 (expedient number: 122831), and ISCIII-REDinREN (RD16/0009 Feder Funds), and developed in the context of AdvanceCat with the support of ACCIÓ (Catalonia Trade \& Investment; Generalitat de Catalunya) under the Catalonian ERDF operational programme (European Regional Development Fund) 2014-2020. FEB is a researcher from Fundació Institut de Recerca en Ciències de la Salut Germans Trias i Pujol, supported by the Health Department of the Catalan Government (Generalitat de Catalunya). Our laboratory is awarded by the Generalitat de Catalunya as Consolidated Research Groups (ref. 2017-SGR-719, 2017-SGR301 and 2017-SGR-483).

\section{Availability of data and materials}

The datasets used and/or analysed during the current study are available from the corresponding authors on request.

\section{Ethics approval and consent to participate}

Clinical grade BM-MSC were produced within the context of four clinical trials (EudraCT Nos. 2010-023998-18, 2010-023999-12, 2013-005025-23 and 2015005786-23). WJ-MSC were isolated from umbilical cord tissue collected within the Concordia Program (EudraCT No. 2015-005786-23). Appropriate donor informed consent for use in research was obtained in all cases.

Consent for publication

Not applicable.

Competing interests

The authors declare that they have no competing interests. 


\section{Author details}

${ }^{1}$ Cell Therapy Service, Blood and Tissue Bank (BST), Barcelona, Catalonia, Spain. ${ }^{2}$ Musculoskeletal Tissue Engineering Group, Vall d'Hebron Research Institute (VHIR) and Universitat Autònoma de Barcelona (UAB), Barcelona, Catalonia, Spain. ${ }^{3}$ REMAR-IVECAT Group, Health Science Research Institute Germans Trias i Pujol (IGTP), Badalona, Catalonia, Spain. ${ }^{4}$ Gene Regulation, Stem Cells and Cancer Program, Centre for Genomic Regulation (CRG), Barcelona, Catalonia, Spain. ${ }^{5}$ Nephrology Service, Germans Trias i Pujol University Hospital, Badalona, Catalonia, Spain. ${ }^{6}$ ICREC Research Program, Health Science Research Institute Germans Trias i Pujol (IGTP), Badalona, Catalonia, Spain. ${ }^{7}$ Medicine Department, Universitat Autònoma de Barcelona (UAB), Badalona, Catalonia, Spain.

Received: 25 July 2019 Revised: 3 October 2019 Accepted: 11 October 2019 Published online: 28 November 2019

\section{References}

1. Im G-I. Clinical use of stem cells in orthopaedics. Eur Cells Mater. 2017;33:183-96.

2. Sampson S, Botto-van Bemden A, Aufiero D. Stem cell therapies for treatment of cartilage and bone disorders: osteoarthritis, avascular necrosis, and non-union fractures. Regen Med. 2015;7(4):S26-32.

3. Naji A, Eitoku M, Favier B, Deschaseaux F, Rouas-Freiss N, Suganuma N. Biological functions of mesenchymal stem cells and clinical implications. Cell Mol Life Sci. 2019; https://doi.org/10.1007/s00018-019-03125-1.

4. Perez JR, Kouroupis D, Li DJ, Best TM, Kaplan L, Correa D. Tissue engineering and cell-based therapies for fractures and bone defects. Front Bioeng Biotechnol. 2018;6(July):1-23.

5. Gómez-Barrena E, Rosset P, Gebhard F, Hernigou P, Baldini N, Rouard H, et al. Feasibility and safety of treating non-unions in tibia, femur and humerus with autologous, expanded, bone marrow-derived mesenchymal stromal cells associated with biphasic calcium phosphate biomaterials in a multicentric, non-comparative trial. Biomaterials. 2019;196:100-8.

6. Giannotti S, Trombi L, Bottai V, Ghilardi M, D'Alessandro D, Danti S, et al. Use of autologous human mesenchymal stromal cell/fibrin clot constructs in upper limb non-unions: long-term assessment. PLoS One. 2013;8(8):e73893.

7. Annamalai RT, Hong X, Schott NG, Tiruchinapally G, Levi B, Stegemann JP. Injectable osteogenic microtissues containing mesenchymal stromal cells conformally fill and repair critical-size defects. Biomaterials. 2019;208:32-44.

8. Prat S, Gallardo-Villares S, Vives M, Carreño A, Caminal M, Oliver-Vila I, et al. Clinical translation of a mesenchymal stromal cell-based therapy developed in a large animal model and two case studies of the treatment of atrophic pseudoarthrosis. J Tissue Eng Regen Med. 2018;12(1):e532-40.

9. Dimmeler S, Leri A. Aging and disease as modifiers of efficacy of cell therapy. Circ Res. 2008;102(11):1319-30.

10. Kretlow JD, Jin Y-Q, Liu W, Zhang WJ, Hong T-H, Zhou G, et al. Donor age and cell passage affects differentiation potential of murine bone marrowderived stem cells. BMC Cell Biol. 2008;9(1):60-72.

11. Saba-Choudhery M, Khan M, Mahmood R, Mehmood A, Khan SN, Riazuddin $\mathrm{S}$. Bone marrow derived mesenchymal stem cells from aged mice have reduced wound healing, angiogenesis, proliferation and anti-apoptosis capabilities. Cell Biol Int. 2012;36(8):747-53.

12. Mendes SC, Tibbe JM, Veenhof M, Bakker K, Both S, Platenburg PP, et al. Bone tissue-engineered implants using human bone marrow stromal cells: effect of culture conditions and donor age. Tissue Eng. 2002;8(6):911-20.

13. Baker N, Boyette LB, Tuan RS. Characterization of bone marrow-derived mesenchymal stem cells in aging. Bone. 2015;70:37-47.

14. Karantalis V, Hernandez-Schulman I, Balkan W, Hare JM. Allogeneic cell therapy: a new paradigm in therapeutics. Circ Res. 2015;116(1):12-5.

15. Mirabel C, Puente-Massaguer E, Del Mazo-Barbara A, Reyes B, Morton P, Gòdia F, et al. Stability enhancement of clinical grade multipotent mesenchymal stromal cell-based products. J Transl Med. 2018;16(1):291-300.

16. Singh-Mohal J, Tailor HD, Khan WS. Sources of adult mesenchymal stem cells and their applicability for musculoskeletal applications. Curr Stem Cell Res Ther. 2012;7(2):103-9.

17. Murphy MB, Moncivais K, Caplan Al. Mesenchymal stem cells: environmentally responsive therapeutics for regenerative medicine. Exp Mol Med. 2013;45(11):e54-16.

18. Dominici M, Le Blanc K, Mueller I, Slaper-Cortenbach I, Marini F, Krause DS, et al. Minimal criteria for defining multipotent mesenchymal stromal cells.
The International Society for Cellular Therapy position statement. Cytotherapy. 2006;8(4):315-7.

19. Guadix JA, Zugaza JL, Gálvez-Martín P. Characteristics, applications and prospects of mesenchymal stem cells in cell therapy. Med Clínica (English Ed). 2017;148(9):408-14.

20. Vives J, Mirabel C. Multipotent Mesenchymal Stromal Cells From Bone Marrow for Current and Potential Clinical Applications. In: Reis RL, Gomes ME, editors. Encyclopedia of Tissue Engineering and Regenerative Medicine, Vol. 2. Academic Press: Elsevier; 2019. p. 503-512. https://doi.org/10.1016/ B978-0-12-801238-3.65506-X.

21. Davies J, Walker J, Keating A. Concise review: Wharton's jelly: the rich, but enigmatic source of mesenchymal stromal cells. Stem Cells Transl Med. 2017;6(7):1620-30.

22. Oliver-Vila I, Coca MI, Grau-Vorster M, Pujals-Fonts N, Caminal M, CasamayorGenescà A, et al. Evaluation of a cell-banking strategy for the production of clinical grade mesenchymal stromal cells from Wharton's jelly. Cytotherapy. 2016;18(1):25-35.

23. La Rocca G, Lo lacono M, Corsello T, Corrao S, Farina F, Anzalone R. Human Wharton's jelly mesenchymal stem cells maintain the expression of key immunomodulatory molecules when subjected to osteogenic, adipogenic and chondrogenic differentiation in vitro: new perspectives for cellular therapy. Curr Stem Cell Res Ther. 2013;8:100-13.

24. Deuse T, Stubbendorf M, Tang-Quan K, Phillips N, Kay MA, Eiermann T, et al. Immunogenicity and immunomodulatory properties of umbilical cord lining mesenchymal stem cells. Cell Transplant. 2011;20(5):655-67. A.

25. Monguió-Tortajada M, Roura S, Gálvez-Montón C, Pujal JM, Aran G, Sanjurjo $L$, et al. Nanosized UCMSC-derived extracellular vesicles but not conditioned medium exclusively inhibit the inflammatory response of stimulated $T$ cells: implications for nanomedicine. Theranostics. 2017;7(2):270-84.

26. Monguió-Tortajada M, Roura S, Gálvez-Montón C, Franquesa M, Bayes-Genis A, Borràs FE. Mesenchymal stem cells induce expression of CD73 in human monocytes in vitro and in a swine model of myocardial infarction in vivo. Front Immunol. 2017;8:1577.

27. Rahman S, Akhtar N, Jamil HM, Banik RS, Asaduzzaman SM. TGF-B/BMP signaling and other molecular events: regulation of osteoblastogenesis and bone formation. 2015;3:15005. https://doi.org/10.1038/boneres.2015.5.

28. Beederman M, Lamplot J, Nan G, Wang J, Liu X, Yin L, et al. BMP signaling in mesenchymal stem cell differentiation and bone formation. J Biomed Sci Eng. 2013;6(8A):32-52.

29. Grafe I, Alexander S, Peterson JR, Snider TN, Levi B, Lee B, et al. TGF- $\beta$ family signaling in mesenchymal differentiation. Cold Spring Harb Perspect Biol. 2017; https://doi.org/10.1101/cshperspect.a022202.

30. Wu M, Chen G, Li YP. TGF- $\beta$ and BMP signaling in osteoblast, skeletal development and bone formation, homeostasis and disease. Bone Res. 2016;4 https://doi.org/10.1038/boneres.2016.9.

31. Cho TJ, Gerstenfeld LC, Einhorn TA. Differential temporal expression of members of the transforming growth factor $\beta$ superfamily during murine fracture healing. J Bone Miner Res. 2002;17(3):513-20.

32. Mehrara B, Most D, Chang J, Bresnick S, Turk A, Schendel S, et al. Basic fibroblast growth factor and transforming growth factor $\beta-1$ expression in the developing dura mater correlates with calvarial bone formation. Plast Reconstr Surg. 1999;104(2):435-44.

33. Miyama K, Yamada G, Yamamoto TS, Takagi C, Miyado K, Sakai M, et al. A BMP-inducible gene, D|x5, regulates osteoblast differentiation and mesoderm induction. Dev Biol. 1999;208(1):123-33.

34. Lee MH, Kim YJ, Kim HJ, Park HD, Kang AR, Kyung HM, et al. BMP-2-induced Runx2 expression is mediated by Dlx5, and TGF- $\beta 1$ opposes the BMP-2induced osteoblast differentiation by suppression of Dlx5 expression. J Biol Chem. 2003;278(36):34387-94.

35. Guerrero F, Herencia C, Almadén Y, Martínez-Moreno JM, Montes De Oca A Rodriguez-Ortiz ME, et al. TGF- $\beta$ prevents phosphate-induced osteogenesis through inhibition of BMP and Wnt/ $\beta$-catenin pathways. PLoS One. 2014;9(2):1-10.

36. Spinella-Jaegle S, Roman-Roman S, Faucheu C, Dunn FW, Kawai S, Galléa S, et al. Opposite effects of bone morphogenetic protein-2 and transforming growth factor- $\beta 1$ on osteoblast differentiation. Bone. 2001;29(4):323-30.

37. Codinach M, Blanco M, Ortega I, Lloret M, Reales L, Coca MI, et al. Design and validation of a consistent and reproducible manufacture process for the production of clinical-grade bone marrow-derived multipotent mesenchymal stromal cells. Cytotherapy. 2016;18(9):1197-208.

38. Oliver-Vila I, Ramírez-Moncayo C, Grau-Vorster M, Marín-Gallén S, Caminal M, Vives J. Optimisation of a potency assay for the assessment of 
immunomodulative potential of clinical grade multipotent mesenchymal stromal cells. Cytotechnology. 2018;70:31-44.

39. Monguió-Tortajada M, Morón-Font M, Gámez-Valero A, Carreras-Planella L, Borràs FE, Franquesa M. Extracellular-vesicle isolation from different biological fluids by size-exclusion chromatography. Curr Protoc Stem Cell Biol. 2019:49(1):e82. https://doi.org/10.1002/cpsc.82. Epub 2019 Jan 30. PubMed PMID: 30698351.

40. Kim DK, Lee J, Kim SR, Choi DS, Yoon YJ, Kim JH, et al. EVpedia: a community web portal for extracellular vesicles research. Bioinformatics. 2015;31(6):933-9.

41. Mathivanan S, Simpson RJ. ExoCarta: a compendium of exosomal proteins and RNA. Proteomics. 2009;9(21):4997-5000.

42. Kalra H, Simpson RJ, Ji H, Aikawa E, Altevogt P, Askenase P, et al. Vesiclepedia: a compendium for extracellular vesicles with continuous community annotation. PLoS Biol. 2012;10(12):8-12.

43. Hsieh J, Fu Y, Chang S, Tsuang Y, Wang H. Mesenchymal stem cells from bone marrow and Wharton's jelly of umbilical cord. Stem Cells and Dev. 2010;19(12):1895-910.

44. Batsali AK, Pontikoglou C, Koutroulakis D, Pavlaki Kl, Damianaki A, Mavroudi I, et al. Differential expression of cell cycle and WNT pathway-related genes accounts for differences in the growth and differentiation potential of Wharton's jelly and bone marrow-derived mesenchymal stem cells. Stem Cell Res Ther. 2017;8:102

45. Hu G, Lee H, Price SM, Shen MM, Abate-Shen C. Msx homeobox genes inhibit differentiation through upregulation of cyclin D1. Development. 2001;128:2373-84

46. Newberry EP, Latifi T, Towler DA. Reciprocal regulation of osteocalcin transcription by the homeodomain proteins Msx2 and Dlx5. Biochemistry. 1998;37:16360-8.

47. Kim Y-J, Lee M-H, Wozney JM, Cho J-Y, Ryoo HM. Bone morphogenetic protein-2-induced alkaline phosphatase expression is stimulated by D|x5 and repressed by Msx2. J Biol Chem. 2004;279(49):50773-80.

48. Shirakabe K, Terasawa K, Miyama K, Shibuya H, Nishida E. Regulation of the activity of the transcription factor Runx2 by two homeobox proteins, Msx2 and Dlx5. Genes Cells. 2001:6(10):851-6.

49. Yingling JM, McMillen WT, Yan L, Huang H, Sawyer JS, Graff J, et al. Preclinical assessment of galunisertib (LY2157299 monohydrate), a first-in-class transforming growth factor- $\beta$ receptor type I inhibitor. Oncotarget. 2018;9(6):6659-77.

50. Ulsamer A, Ortuño MJ, Ruiz S, Susperregui ARG, Osses N, Rosa JL, et al. BMP2 induces osterix expression through up-regulation of Dlx 5 and its phosphorylation by p38. J Biol Chem. 2008;283(7):3816-26.

51. Kim HJ, Kim SH. Tanshinone IIA enhances BMP-2-stimulated commitment of C2C12 cells into osteoblasts via p38 activation. Amino Acids. 2010;39(5):1217-26.

52. Heo JS, Lee SG, Kim HO. Distal-less homeobox 5 is a master regulator of the osteogenesis of human mesenchymal stem cells. Int J Mol Med. 2017:40(5):1486-94.

53. Blanco JF, Villarón EM, Pescador D, Da Casa C, Gómez V, Redondo AM, et al. Autologous mesenchymal stromal cells embedded in tricalcium phosphate for posterolateral spinal fusion: results of a prospective phase I/I clinical trial with long-term follow-up. Stem Cell Res Ther. 2019;10(63):1-8.

54. Kaigler D, Pagni G, Ho Park C, Braun TM, Holman LA, Yi E, et al. Stem cell therapy for craniofacial bone regeneration: a randomized, controlled feasibility trial. Cell Transplant. 2013;22(5):767-77.

55. Liebergall M, Schroeder J, Mosheiff R, Gazit Z, Yoram Z, Rasooly L, et al. Stem cell-based therapy for prevention of delayed fracture union: a randomized and prospective preliminary study. Mol Ther. 2013;21(8):1631-8.

56. Caminal M, Vélez R, Rabanal RM, Vivas D, Batlle-Morera L, Aguirre M, et al. A reproducible method for the isolation and expansion of ovine mesenchymal stromal cells from bone marrow for use in regenerative medicine preclinical studies. J Tissue Eng Regen Med. 2017;11(12):3408-16.

57. Roura S, Vives J. Extracellular vesicles: Squeezing every drop of regenerative potential of umbilical cord blood. Metabolism. 2019;95:102-104. https://doi. org/10.1016/j.metabol.2019.02.006. Epub 2019 Mar 1. PubMed PMID: 30831143.

58. Roura S, Bayes-Genis A. Toward standardization of mesenchymal stromal cell-derived extracellular vesicles for therapeutic use: a call for action. Proteomics. 2019;19:1800397.

59. Zhou Y, Yamamoto Y, Ochiya T, Xiao Z, Itaya T. Distinct Mirna expression patterns of extracellular vesicles derived from 4 types of mesenchymal stem cells. J Stem Cell Res Ther. 2018;8:415. https://doi.org/10.4172/2157-7633. 1000415.

60. Wang X, Omar O, Vazirisani F, Thomsen P, Ekström K. Mesenchymal stem cell-derived exosomes have altered microRNA profiles and induce osteogenic differentiation depending on the stage of differentiation. PLoS One. 2018;13(2):e0193059.
61. Izu Y, Ezura Y, Koch M, Birk DE, Noda M. Collagens VI and XII form complexes mediating osteoblast interactions during osteogenesis. Cell Tissue Res. 2016;364(3):623-35.

\section{Publisher's Note}

Springer Nature remains neutral with regard to jurisdictional claims in published maps and institutional affiliations.
Ready to submit your research? Choose BMC and benefit from:

- fast, convenient online submission

- thorough peer review by experienced researchers in your field

- rapid publication on acceptance

- support for research data, including large and complex data types

- gold Open Access which fosters wider collaboration and increased citations

- maximum visibility for your research: over $100 \mathrm{M}$ website views per year

At BMC, research is always in progress.

Learn more biomedcentral.com/submissions 